\title{
The Parallel Algorithm Based on Genetic Algorithm for Improving the Performance of Cognitive Radio
}

\author{
Liu Miao $\mathbb{D}^{1}{ }^{1}$ Zhenxing Sun, $^{1,2,3}$ and Zhang Jie ${ }^{4}$ \\ ${ }^{1}$ Department of Electronics and Information Engineering, Northeast Petroleum University, No. 550, West Hebei Avenue, \\ Qinhuangdao 066004, China \\ ${ }^{2}$ School of Computer Science and Engineering, Northeastern University, Shenyang, China \\ ${ }^{3}$ Qinhuangdao Economic and Technological Development Zone, No. 143 Taishan Road, Qinhuangdao, Hebei 066004, China \\ ${ }^{4}$ Editorial Board of Journal of Jilin University (Information and Science Edition), Jilin University, Changchun 130012, China \\ Correspondence should be addressed to Liu Miao; lm_jlu@163.com
}

Received 21 September 2017; Revised 20 December 2017; Accepted 4 January 2018; Published 12 March 2018

Academic Editor: Fei Hu

Copyright (C) 2018 Liu Miao et al. This is an open access article distributed under the Creative Commons Attribution License, which permits unrestricted use, distribution, and reproduction in any medium, provided the original work is properly cited.

\begin{abstract}
The intercarrier interference (ICI) problem of cognitive radio (CR) is severe. In this paper, the machine learning algorithm is used to obtain the optimal interference subcarriers of an unlicensed user (un-LU). Masking the optimal interference subcarriers can suppress the ICI of CR. Moreover, the parallel ICI suppression algorithm is designed to improve the calculation speed and meet the practical requirement of CR. Simulation results show that the data transmission rate threshold of un-LU can be set, the data transmission quality of un-LU can be ensured, the ICI of a licensed user (LU) is suppressed, and the bit error rate (BER) performance of LU is improved by implementing the parallel suppression algorithm. The ICI problem of CR is solved well by the new machine learning algorithm. The computing performance of the algorithm is improved by designing a new parallel structure and the communication performance of CR is enhanced.
\end{abstract}

\section{Introduction}

Cognitive radio (CR) technologies based on the dynamic spectrum access concept can utilize temporarily idle spectrum resources $[1,2]$. CR can improve the current tension of spectrum resources and enhance wireless communication performance [3]. Spectrum Pooling is a very effective spectrum utilization technology of CR. The licensed spectrum of different businesses is merged into a public spectrum pool by Spectrum Pooling [4]. Unlicensed users can utilize the idle spectrum which is not occupied by licensed users in Spectrum Pooling. Spectrum Pooling based on Orthogonal Frequency Division Multiplexing (OFDM) has been widely accepted [5]. However, the side lobe interference of OFDM signal is the shortcoming of Spectrum Pooling based on OFDM [6]. Spectrum Pooling based on Wavelet-Based Orthogonal Frequency Division Multiplexing (SP-WOFDM) can not only obtain the same perfect performance of OFDM but also configure subchannels flexibly and suppress Intersymbol Interference (ISI), pulse interference, and narrow band interference more effectively. And it can realize multirate signal transmission to meet the requirements of different businesses and business quality more conveniently $[7,8]$. Moreover, SP-WOFDM owns better bandwidth effectiveness without side lobe interference because it does not need guard interval and pilot.

In a multipath environment, the orthogonality of WOFDM signal is lost so that Intersymbol Interference (ISI) and Intercarrier Interference (ICI) occur $[9,10]$. The performance of SP-WOFDM can be destroyed by ICI and ISI. Masking one or more un-LU's subcarriers can mitigate the ICI of SPWOFDM. But it can sacrifice bandwidth in the rental system. So, it is important to research the trade-off between reducing the interference power and maintaining the bandwidth of the unlicensed system. The genetic algorithm (GA) is an efficient, practical, and robust optimization technique. Its essence is parallel, efficient, and global search methods. It can obtain and accumulate the related knowledge of the search space automatically and control the search process adaptively to get the optimal solution. The GA has characteristics including 
operating on the encoding of the parameter, no deducing and additional information, the uncertainty of optimization rules, self-organization, self-adaption, and self-learning, compared with the traditional optimal algorithms.

In the paper, GA for suppression ICI energy of SPWOFDM is proposed. The optimal interference subcarriers for suppression ICI that the un-LU causes to the LU can be obtained by a new algorithm. Moreover, the parallel ICI suppression algorithm is designed to improve the calculation speed of ICI suppression based on GA and meet the practical requirement of $\mathrm{CR}$. The new algorithm does not only realize the trade-off between reducing the interference power and maintaining the bandwidth of the unlicensed system, but also consider the calculation speed and practicability of the algorithm.

The paper is organized as follows. The system model and ICI energy of SP-WOFDM are provided in Section 2. In Section 3, GA for ICI suppression is proposed. The parallel ICI suppression algorithm is designed in Section 4. The simulation results are described in Section 5. Section 6 concludes the paper.

\section{The CR System Model}

The SP-WOFDM with an ICI suppression unit is shown in Figure 1 . The modulated signal $y(n)$ can be expressed in the discrete domain as [11]

$$
y(n)=\sum_{i} \sum_{j}^{M-1}\left(\bar{x}_{i, j} h_{j}(n-i M)\right) \uparrow_{M} \quad \bar{x}_{i, j}=w_{i, j} x_{i, j} .
$$

In Spectrum Pooling, the average ICI energy which influences subcarrier $j$ of LU can be deduced as [12]

$$
\begin{aligned}
& \sigma_{R U_{j}}^{2} \\
& \quad=\frac{1}{N_{l}} \sum_{i=1}^{N_{l}}\left(\sum_{n=-\infty}^{+\infty}\left|\sum_{m=-\infty}^{+\infty} h_{l_{i}}(m-p) h_{r_{j}}^{*}\left(m-2^{D_{i}} n\right)\right|^{2}\right) .
\end{aligned}
$$

\section{The GA for CR ICI Suppression}

GA is an adaptive method to solve the search problem. It is based on parallel search of the chromosome group, selecting operations with guessing, switching operations, and mutation operations. So, GA has the following characteristics.

GA begins its search from the set of problem solutions, rather than the single solution. This is a great difference between GA and traditional optimization algorithms. The traditional optimization algorithms get local optimal solutions easily because they obtain the optimal solution from a single initial value iteration. GA begins its search from the set of problem solutions. So, it covers a wide area and it is good for global choice.

GA need not search spatial knowledge or other auxiliary information. It utilizes the fitness function value to evaluate individuals and carry out genetic operation. The fitness function is not limited to continuous differentiable functions, and its definition domain can be set arbitrarily. This feature expands the application range of GA greatly.
GA adopts the changing rules of probability rather than deterministic rules to guide its search direction.

GA is self-organizing, adaptive, and self-learning. When GA uses the information of the evolution process to organize the search, the individual with large fitness has a higher probability of survival and can obtain a more adaptive genetic structure.

The Spectrum Pooling ICI suppression algorithm based on GA is shown in Figure 2. At first, the data transmission rate threshold $R_{\text {thr }}$ of un-LU and the fitness threshold are set. The masked subcarrier sequence length $L$ is calculated by $R_{\mathrm{thr}}$. The subcarrier set $b_{i}, i=1,2, \ldots, L$, is randomly selected from un-LU subcarriers as the original sequence. The better intermediate carrier sequence is selected by the fitness function. The new subcarrier sequence is produced by overlapping and variation. The fitness function is adjusted constantly to produce the new intermediate carrier sequence until the fitness reaches the set threshold. The optimal interference subcarrier sequence is obtained. The CR interference suppression is realized by masking the optimal interference subcarrier sequence.

The specific process of ICI suppression algorithm is described as follows:

(1) Setting the data transmission rate threshold $R_{\text {thr }}$ of unLU and the fitness threshold.

(2) Original selecting subcarrier sequence of un-LU.

We select subcarrier set $b_{i}, i=1,2, \ldots, L$, from subcarriers of un-LU randomly,

$$
L=\frac{R(x)-R_{\mathrm{thr}}}{\text { bit }_{x}},
$$

where $R(x)$ is the original data transmission rate of un-LU before the interference is suppressed. bit $x_{x}$ is the number of bits assigned to each subcarrier.

(3) Selecting a better intermediate carrier sequence.

A better intermediate carrier sequence is selected by the fitness function. The average ICI energy of the $j$ un-LU's subcarrier infecting LU (2) is selected as the fitness function of the $j$ un-LU's subcarrier. The fitness function can be described as follows:

$$
\begin{aligned}
& f\left(b_{j}\right) \\
& =\frac{1}{N_{l}} \sum_{i=1}^{N_{l}}\left(\sum_{n=-\infty}^{+\infty}\left|\sum_{m=-\infty}^{+\infty} h_{l_{i}}(m-p) h_{r_{j}}^{*}\left(m-2^{D_{i}} n\right)\right|^{2}\right),
\end{aligned}
$$

where $h_{l}$ is the filter of LU for modulating subcarriers, $h_{l}=\left[h_{l_{1}} h_{l_{2}} \cdots h_{l_{N_{l}}}\right] . h_{r}$ is the filter of un-LU for modulating subcarriers, $h_{r}=\left[h_{r_{1}} h_{r_{2}} \cdots h_{r_{N r}}\right]$. The ranges of $m$ and $n$ are decided by the length of the filter. $p$ is the additional delay of the channel. $h_{l_{i}}$ and $h_{r_{j}}$ are wavelet filter functions for modulating subcarriers $i$ and $j$ of LU, respectively. $N_{l}$ is the number of LU's subcarriers. $D_{i}, i=0 \cdots N-1$, is the level of filter $i$.

The fitness adjustment formula can be described as

$$
\begin{aligned}
& f\left(b_{j}+1\right)=a f\left(b_{j}\right)+b, \\
& \max \left\{f\left(b_{j}\right) \mid b_{j}\{0,1\} L\right\},
\end{aligned}
$$




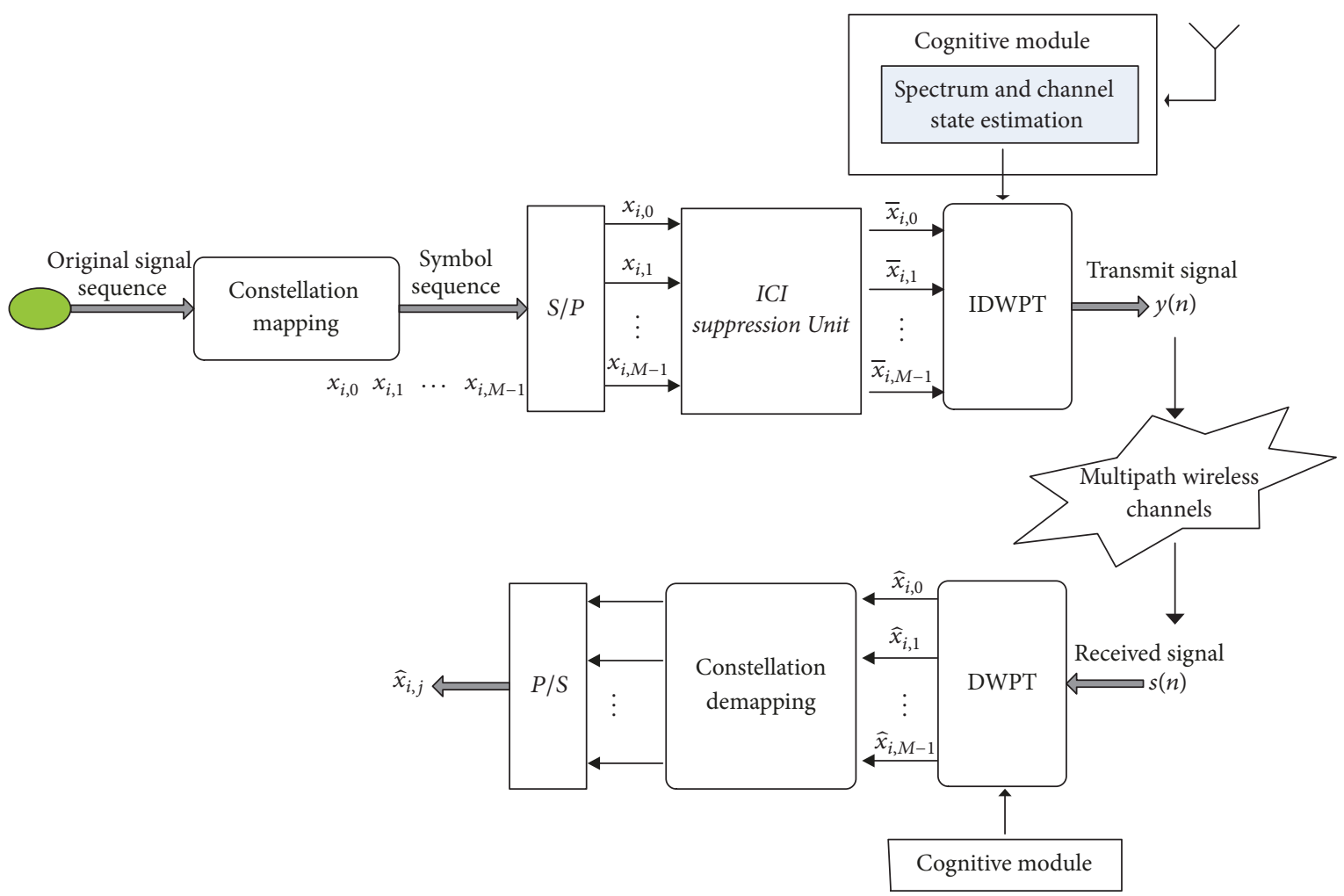

FIGURE 1: The block diagram of Spectrum Pooling with an ICI suppression unit.

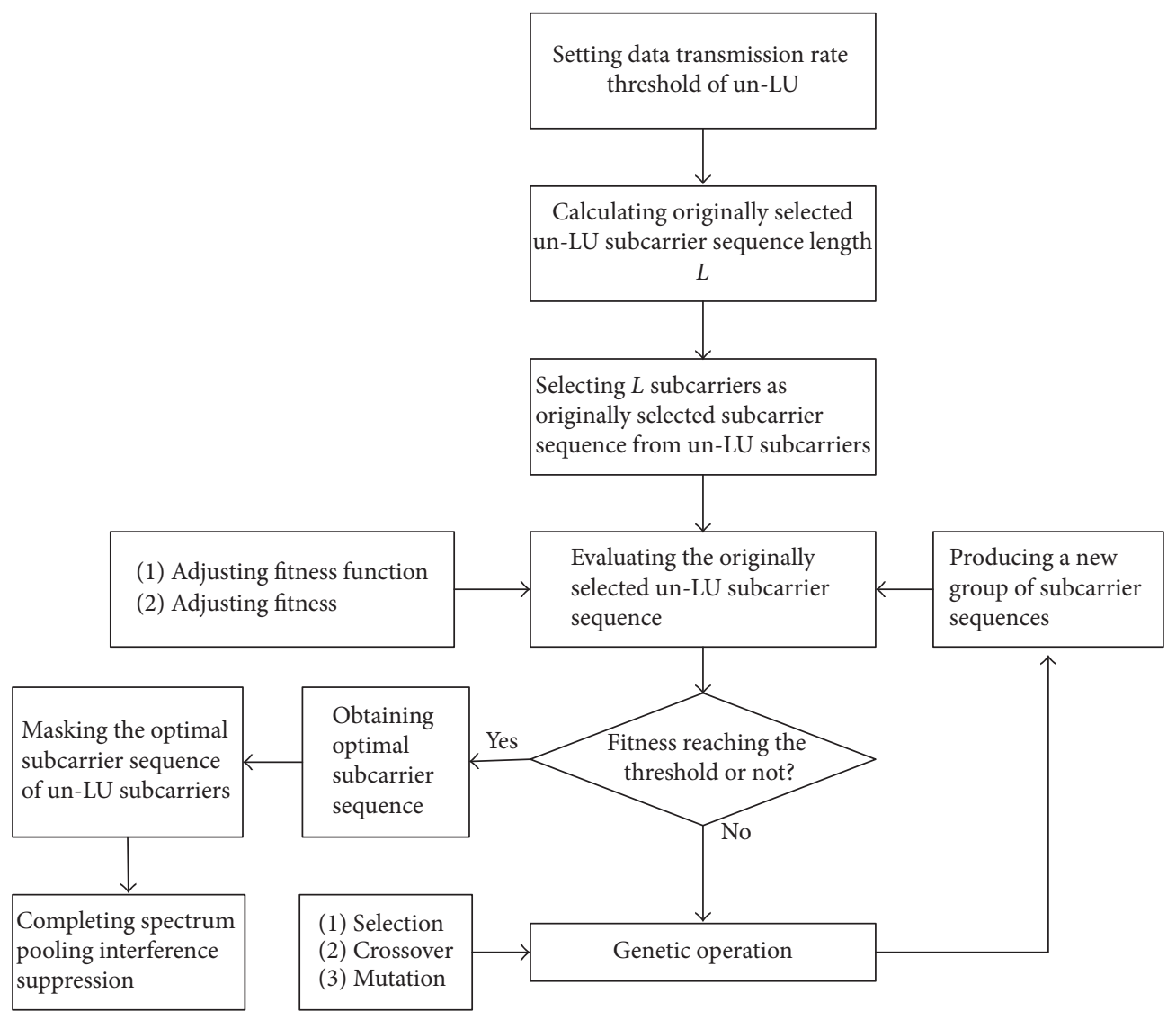

FIGURE 2: The flowchart of GA for ICI suppression. 
where $f\left(b_{j}\right) \neq f\left(b_{j}+1\right), a$ and $b$ are the fitness parameters, and $L$ is the length of the original set.

(4) Crossing and generating the new optional carriers.

Two intermediate carriers selected from intermediate carrier sequence are crossed to generate the new optional carriers by crossover probability $p \cdot p$ is defined as

$$
p\left(b_{j}\right)=\frac{f\left(b_{j}\right)}{\sum_{j=1}^{n} f\left(b_{j}\right)},
$$

where $b_{j}$ is the original carrier sequence and $f\left(b_{j}\right)$ is a fitness function.

(5) Variation and generating the new optional carriers.

The new optional carriers can be generated by variation probability $p_{m} \cdot p_{m}$ is defined as follows.

For a given carrier group $s=b_{1} b_{2} \cdots b_{L}$,

$$
O\left(p_{m}, x\right): b_{j}= \begin{cases}1-b_{j}, & \text { if } x_{j} \leq p_{m} \\ b_{j}, & \text { else, }\end{cases}
$$

where $j \in\{1,2, \ldots, L\}, L$ is the original carrier set, and $x_{j} \in$ $[1,0]$.

(6) Obtaining optimal interference subcarrier sequences.

When the fitness of optimal subcarrier reaches the given threshold, the optimal interference subcarrier sequence is obtained. The interference suppression of CR is realized by masking the optimal interference subcarrier sequence.

The complexity of GA is $O\left(N^{2}\right)$.

\section{Parallel Algorithm Design for CR}

Parallel computing is the process of using multiple computing resources to solve computing problems. Traditional serial computing is the process of performing single operations one by one on a single central processing unit. But parallel computing can simultaneously perform multiple operations on a set of processor units. Parallel computing can quickly solve large and complex computing problems. In the multicore era, better performance can be obtained by designing a parallel computing algorithm based on multithreads on a multicore computing platform.

The Spectrum Pooling ICI suppression parallel algorithm based on multithreads is designed by us to improve the calculation speed of Spectrum Pooling ICI suppression algorithm based on GA and make it more practical. The Spectrum Pooling ICI suppression algorithm based on GA can be parallelized as follows:

(1) Parallelizing fitness evaluation of originally selected subcarriers.

The fitness evaluation of the selected subcarriers takes up most of the execution time and there is no dependence on each other. So, fitness evaluation computing of originally selected subcarriers can be assigned to different cores in a multicore processor.

(2) Parallelizing each genetic operator.

The operation of cross, selection, and variation can be threaded and performed on different cores independently and concurrently.
The specific process of parallel ICI suppression algorithm is described as follows:

(1) Setting the data transmission rate threshold of un-LU and the fitness threshold.

(2) Original selecting of subcarrier sequence of un-LU.

(3) Parallel selecting of better intermediate carrier sequence.

(4) Parallel performing of the crossing operation and generating the new optional carriers.

(5) Parallel performing of the variation operation and generating the new optional carriers.

(6) Obtaining optimal interference subcarrier sequences.

The parallel ICI suppression algorithm can run on a multicore computing platform and it has the advantage of multithreads. So, the parallel ICI suppression algorithm can not only improve the calculation speed of the original ICI suppression algorithm based on GA, but also meet the practical requirement of $C R$.

\section{Simulation Results}

To test the performance of the ICI suppression algorithm based on GA, we construct Spectrum Pooling with 32 subcarriers. Spectrum Pooling is divided into 8 subbands and each subband matches 4 subcarriers. The Daubechies-4 ( $\mathrm{Db}(4))$ is implemented to modulate the subcarriers of Spectrum Pooling. QPSK is used to realize symbol constellation.

To simulate the wireless channel environment, we use the two-path wireless channel model. The impulse response of channel is defined as follows [13]:

$$
h_{\mathrm{ch}}(n)=\delta(n)+\delta(n-p p) e^{j \theta_{1}},
$$

where $p p$ is a positive integer; it represents an additional delay of the channel and is normalized as a symbol period. $\theta_{1}$ is the random phase; the range of $\theta_{1}$ is $[0,2 \pi)$.

Set the crossover probability $p=0.8$ and variation probability $p_{m}=0.1$.

The simulation scene is designed as follows.

Scene 1. LU occupies the central 3-6 subbands and 4-5 subbands of Spectrum Pooling, respectively.

Scene 2. LU occupies the 2, 3, 5, and 7 subbands of Spectrum Pooling. LU occupies the 2, 4, 6, and 8 subbands of Spectrum Pooling. LU occupies the 2 and 6 subbands of Spectrum Pooling.

Figures 3 and 4 are the simulation results of Scene 1 and Scene 2. It is shown that the ICI energy of un-LU decreases if the number of masked optimal interference subcarriers increases for both Scene 1 and Scene 2. Furthermore, the ICI energy of un-LU decreases with the number of LU's subcarriers increasing if the same number of optimal interference subcarriers is masked.

In Figures 5 and 6 , the LU occupied 8 subcarriers in the Spectrum Pooling and the two-path wireless channel model 


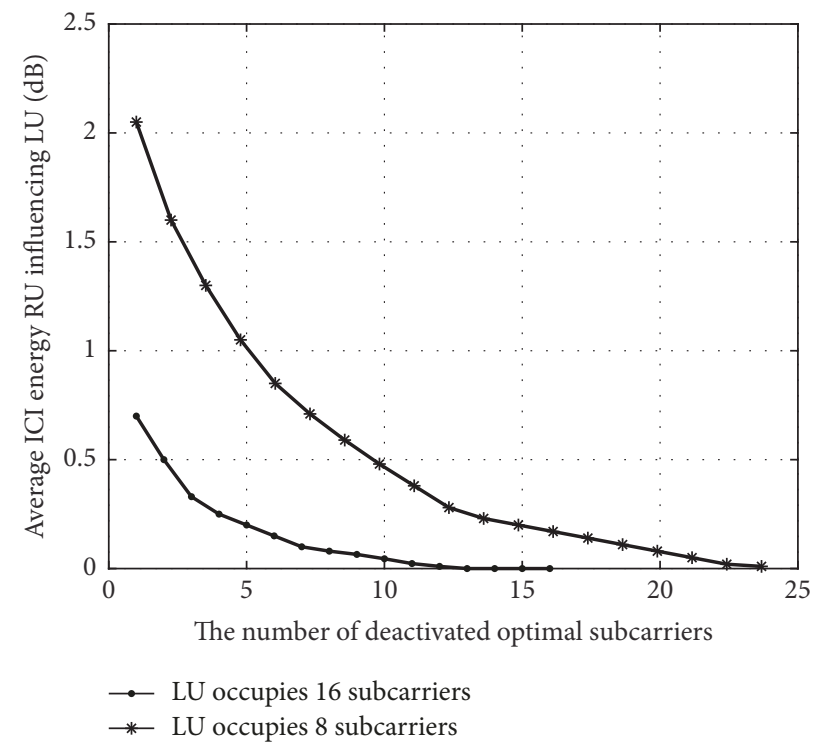

FIGURE 3: The relation between the number of masked optimal subcarriers and ICI energy in Scene 1 based on $\mathrm{Db}(4)$.

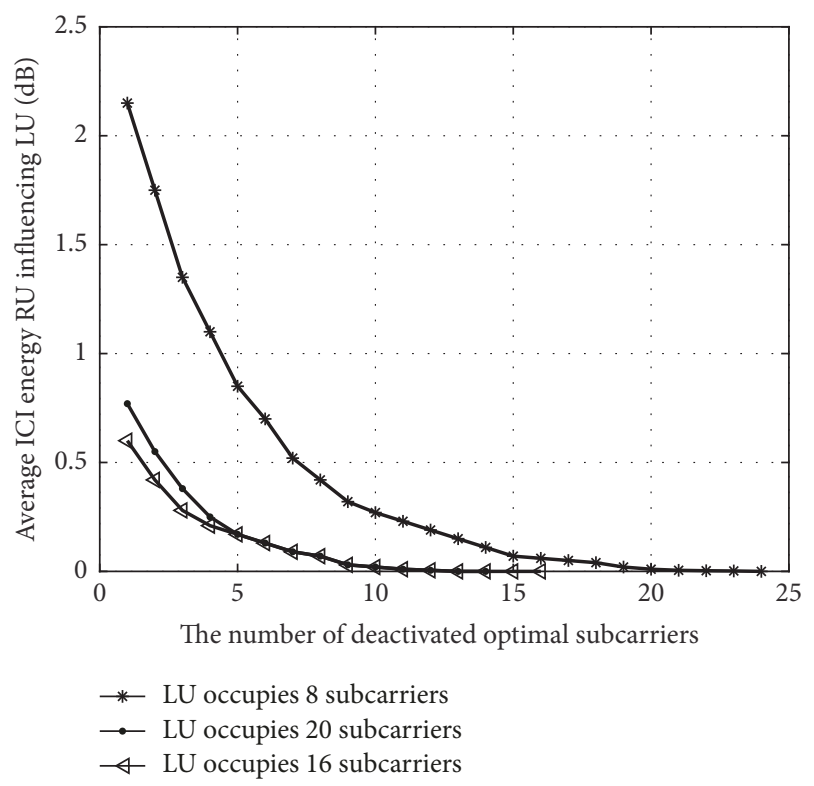

FIgURE 4: The relation between the number of masked optimal interference subcarriers and ICI energy in Scene 2 based on Db(4).

with $p=0, \theta_{1}=\pi / 5$ is implemented. In the wireless channel with only multipath effect, Figures 5 and 6 are the simulation results of LU occupying central successive subbands and the 2 and 6 subbands. It is shown that the BER performance of $\mathrm{LU}$ is improved if the number of masked optimal interference subcarriers increases.

The GA is used to obtain the optimal subcarriers. Figures $3,4,5$, and 6 describe the ICI performance of LU masking different numbers of optimal subcarriers and the BER performance of LU masking different numbers of optimal subcarriers. The results show that the ICI of LU can be suppressed and BER performance of $\mathrm{LU}$ can be improved by GA.

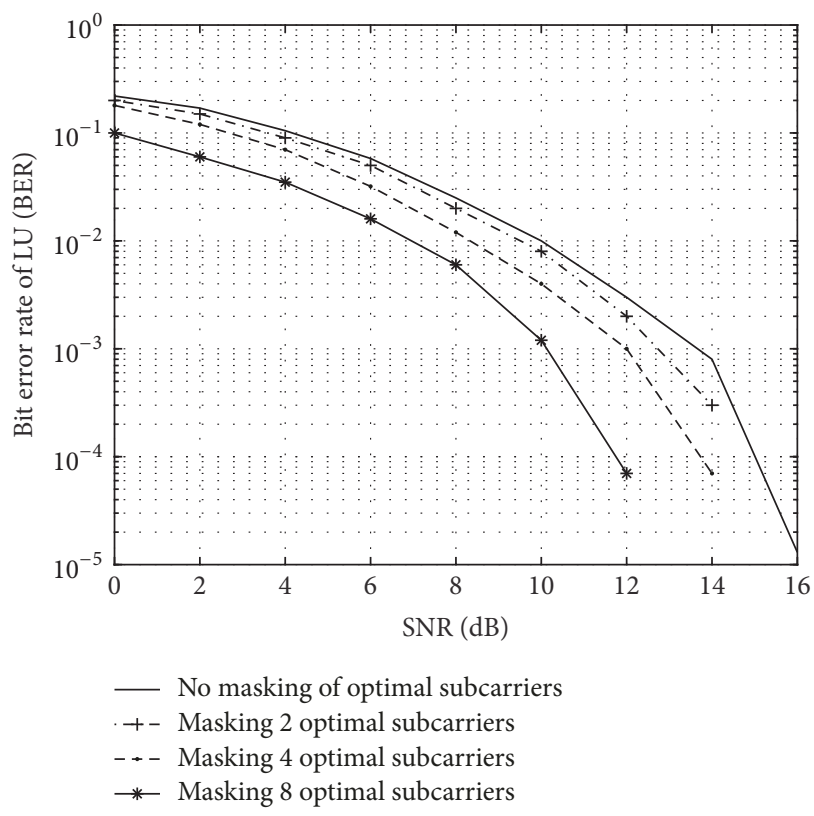

FIGURE 5: $p=0, \theta_{1}=\pi / 5$, and BER performance of LU occupying central successive subbands based on $\mathrm{Db}(4)$.

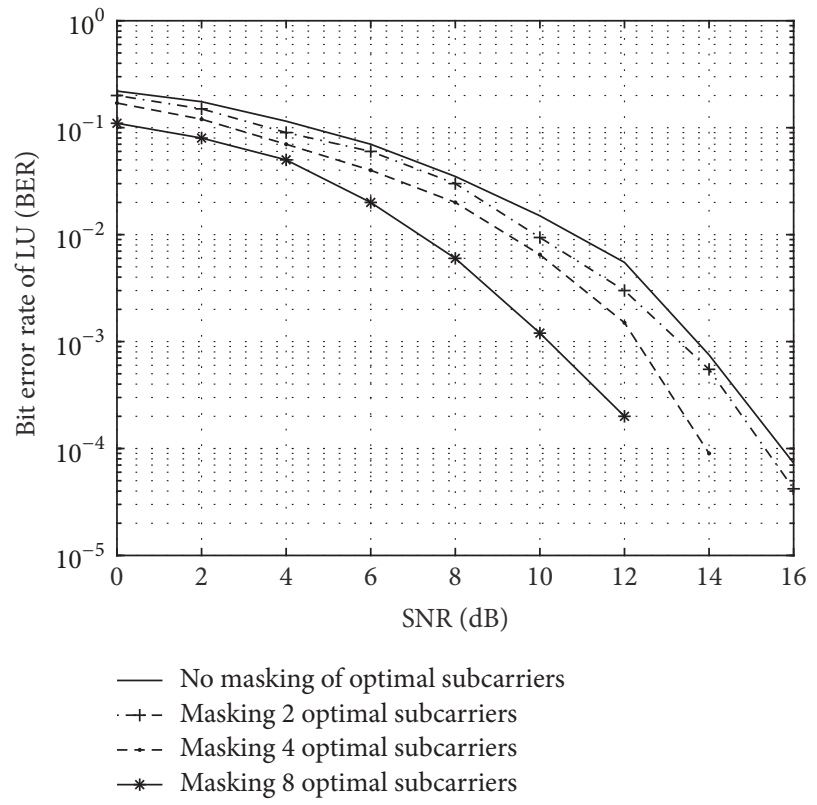

FIGURE 6: $p=0, \theta_{1}=\pi / 5$, and BER performance of LU occupying 2 and 6 subbands based on $\mathrm{Db}(4)$.

\section{Conclusions}

The CR is a new method to share spectrum resources with more flexibility and efficiency. However, the orthogonality of LU signal and un-LU signal is disrupted because of multipath fading impulse. So, ICI between LU and un-LU occurs. GA can obtain and accumulate the related knowledge of search space automatically and control the search process adaptively to get the optimal solution. This paper proposes and discusses the GA used to obtain the optimal interference subcarriers 
for ICI suppression. The average ICI energy of the $j$ un-LU's subcarrier infecting $\mathrm{LU}$ is selected as the fitness function of the $j$ un-LU's subcarrier.

Parallelizing the original algorithm is an effective method to improve the operation speed. So, the parallel ICI suppression algorithm is designed. The simulation results show that the average ICI power of LU can be improved by masking the optimal interference subcarriers. In addition, the BER performance of LU is improved. In the future, more selflearning optimization algorithms will be studied to obtain optimal interference subcarriers for suppressing the ICI of Spectrum Pooling.

\section{Conflicts of Interest}

The authors declare that they have no conflicts of interest.

\section{Acknowledgments}

This work was supported by the National Natural Science Foundation of China (no. 61601111) and Postdoctoral Scientific Research Developmental Fund of China (no. LBHQ16309).

\section{References}

[1] Z. I. A. M. Ahmed, K. H. D. Bilal, and D. M. M. Alhassan, "Cognitive radio network review," International Journal of Engineering, vol. 13, no. 1, pp. 320-327, 2016.

[2] A. A. Khan, M. H. Rehmani, and M. Reisslein, "Cognitive radio for smart grids: survey of architectures, spectrum sensing mechanisms, and networking protocols," IEEE Communications Surveys \& Tutorials, vol. 18, no. 1, pp. 860-898, 2016.

[3] M. R. Mili and L. Musavian, "Interference efficiency: a new concept to analyze the performance of cognitive radio networks," in Proceedings of the 2017 IEEE International Conference on Communications Workshops, ICC Workshops 2017, pp. 1105-1110, France, May 2017.

[4] B. Cao, Q. Zhang, and J. W. Mark, Optimal Communication Strategies in Cooperative Cognitive Radio Networking. Cooperative Cognitive Radio Networking, Springer International Publishing, 2016.

[5] K. V. Krishnan, R. M. Sajith, and S. Khara, "Dynamic resource allocation in OFDM based cognitive radio system considering primary user QoS and secondary user proportional constraints," Journal of Communications Technology and Electronics, vol. 60, no. 11, pp. 1269-1275, 2015.

[6] G. Singh and P. Mehta, "Implementation of optimization algorithm by sub-carriers assignment and power allocation for LTE and cognitive radio network using OFDM signal," European Journal of Advances in Engineering and Technology, vol. 2, no. 3, pp. 19-23, 2015.

[7] D. Shahbaztabar and H. Farrokhi, "Designing non-contiguous orthogonal frequency division multiplexing transceiver based on wavelet transform and removable cyclic prefix for spectrum sharing in cognitive radio systems," IET Communications, vol. 8, no. 9, pp. 1508-1517, 2014.

[8] M. Liu, K. Wang, Y. Huang, and X. Li, "Reducing PAPR by selecting optimal wavelet tree structure in WOFDM," Computers and Electrical Engineering, vol. 37, no. 3, pp. 253-260, 2011.
[9] J. Chunkath, S. Arjun, V. Sheeba, and S. A. Raj, "Performance improvement of multicarrier systems using wavelet filter banks," Procedia Technology, vol. 24, pp. 775-781, 2016.

[10] N. Taşpinar and Y. T. Bozkurt, "PAPR reduction using genetic algorithm in lifting-based wavelet packet modulation systems," Turkish Journal of Electrical Engineering \& Computer Sciences, vol. 24, no. 1, pp. 184-195, 2016.

[11] L. Miao, W. Ke, and H. Yan, "Adaptive guard bands for Spectrum Pooling," in Proceedings of the 2010 2nd International Conference on Computer Engineering and Technology, ICCET 2010, pp. V4103-V4107, China, April 2010.

[12] L. Miao, L. Na, and Z.-X. Sun, "Optimal deactivated sub-carriers guard bands for spectrum pooling systems based on wavelet-based orthogonal frequency division multiplexing," IET Communications, vol. 8, no. 7, pp. 1117-1123, 2014.

[13] B. G. Negash and H. Nikookar, "Wavelet-based multicarrier transmission over multipath wireless channels," IEEE Electronics Letters, vol. 36, no. 21, pp. 1787-1788, 2000. 


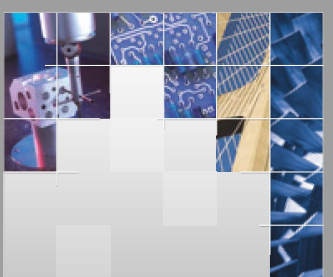

\section{Enfincering}
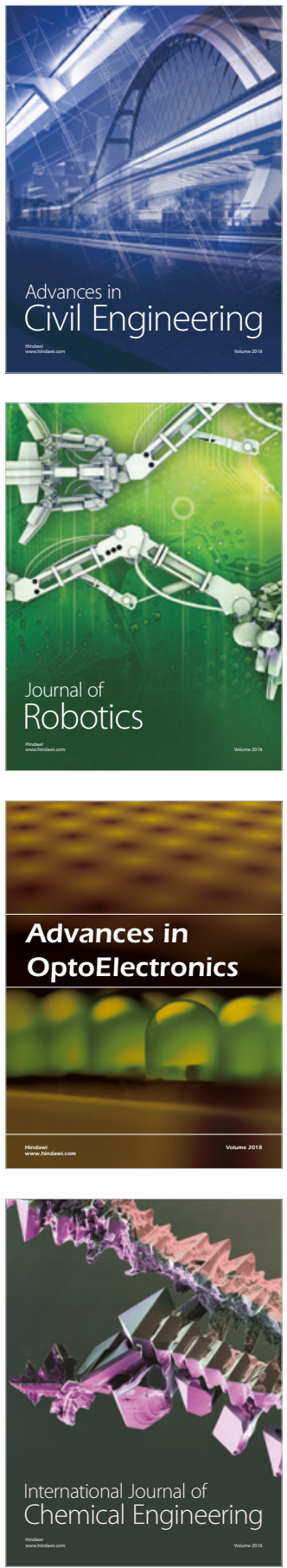

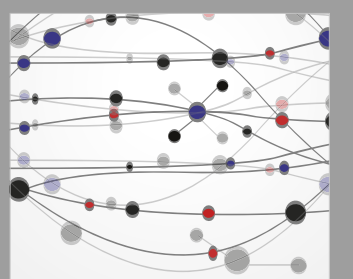

\section{Rotating \\ Machinery}

The Scientific World Journal

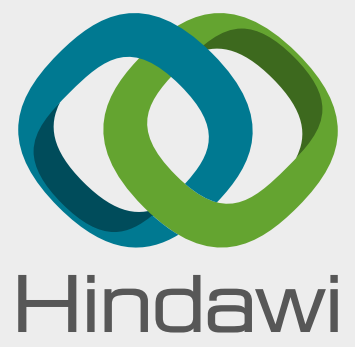

Submit your manuscripts at

www.hindawi.com
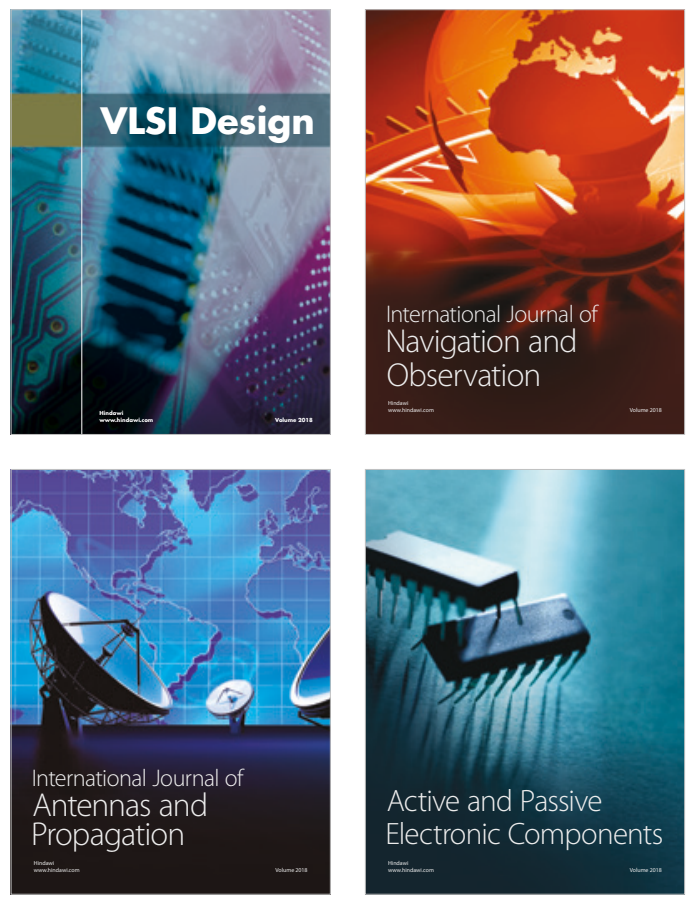
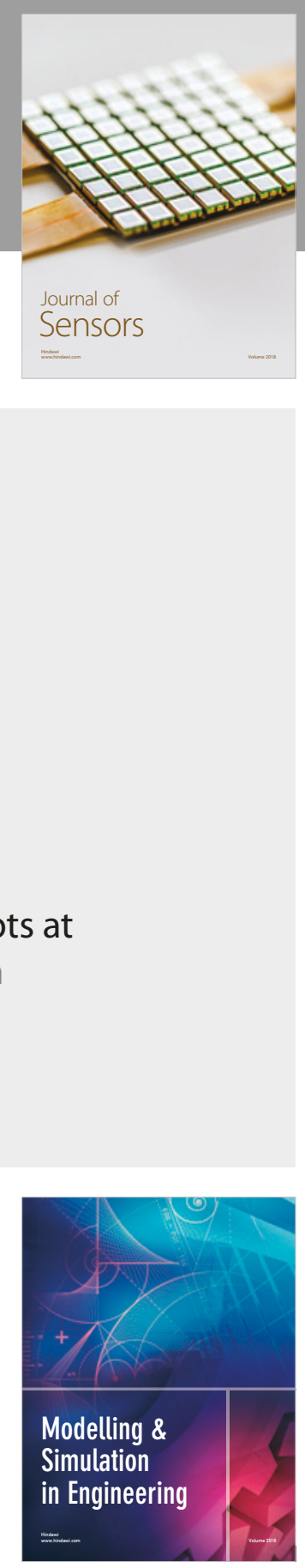

\section{Advances \\ Multimedia}
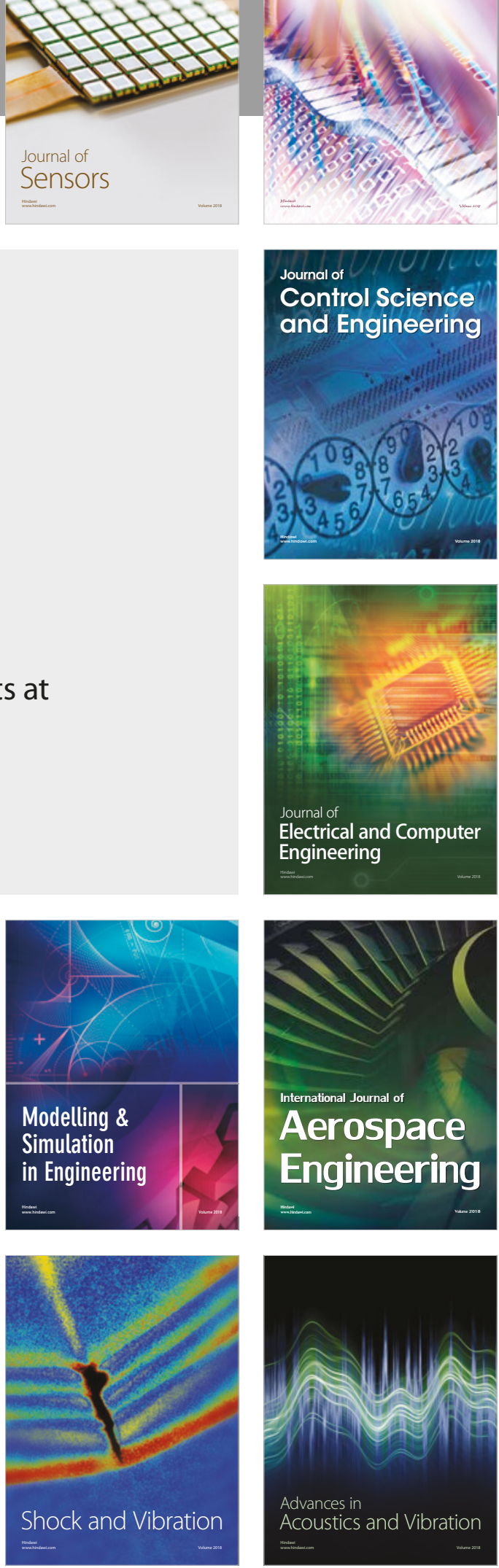